\title{
La automedicación entre los profesionales de la salud en Fátima/Bahia
}

\author{
Self-medication among health professionals in the municipality of Fátima/BA \\ Automedicação entre profissionais de saúde do município de Fátima/BA
}

Wellington Pereira Rodrigues ${ }^{1 *}$, Priscila Dantas Gonçalves ${ }^{2}$, lara de Jesus Carvalho², Kaenne Souza de Santana ${ }^{2}$, Valquíria Ribeiro de Santana ${ }^{2}$, Ana Paula Gomes Soares ${ }^{3}$, Ingrid Borges Siqueira ${ }^{4}$, Leonardo Fabrício Gomes Soares ${ }^{5}$, Cínthia Caldas Rios Soares ${ }^{6}$, Renan Sallazar Ferreira Pereira ${ }^{7}$.

\section{RESUMEN}

Objetivo: Alertar al público sobre el uso racional de los medicamentos y de los factores asociados con esta práctica, ressaltando la importância de un profesional de la salud y promoviendo acciones preventivas. Método: Es un estúdio descriptivo, de carácter exploratório y con sección transversal. Fueron realizados estudios en campo de carácter cuantitativo, en la unidad de Urgencias y de Maternidad Maria Jovita do Nascimento, en cinco unidad básica de salud, en al los trabajadores sanitarios de la comunidad Programa, en el Centro de Vacunas y en la Vigilancia Epidemiológica en Fátima - BA. Los datos fueron procedentes de cuestionarios semi-estructurados para entender las opiniones individuales sobre el tema en questión. El estudio fue aprobado por el Comité de Ética e Investigación con Seres Humanos del Centro Universitario AGES bajo el dictamen oㅡ 024-2018. Resultados: De los 45 (100\%) profesionales, (16; 35,5\%) eran enfermeiros, $(26 ; 57,8 \%)$ eran técnicos de enfermeira y $(3 ; 6,7 \%)$ eran auxiliares de enfermería. La mayor prevalencia de automedicación fue entre los técnicos de enfermería $(5 ; 19,2 \%)$ y de los enfermeros $(4 ; 25 \%)$. Todos los profesionales $(45 ; 100 \%)$ son consientes de los riesgos de la automedicación. El fármaco más utilizado por $(14 ; 31,1 \%)$ de los profesionales en los últimos 10 días fue dipirona. Conclusión: Esto indica que hay uma alta tasa de automedicación entre los profesionales de la salud.

Palabras-claves: Automedicación, Medicamentos, Trabajadores.

\begin{abstract}
Objective: Alert the public about the rational use of medicines and the factors associated with this practice, highlighting the importance of a health professional and promoting preventive actions. Method: It is a descriptive study, exploratory character and cross section. Quantitative field studies were carried out in the Maria Jovita do Nascimento Emergency and Maternity unit, in five basic health units, in the health workers of the Program community, in the Vaccine Center and in the Epidemiological Surveillance in Fatima - BA. The data came from semi-structured questionnaires to understand the individual opinions on the subject in question. The study was approved by the Ethics and Research Committee with Human Beings of the AGES University Center under Opinion No. 024-2018. Results: Of the 45 (100\%) professionals, (16, 35.5\%) were nurses, $(26,57.8 \%)$ were nursing technicians and $(3,6.7 \%)$ were nursing assistants. The highest prevalence
\end{abstract}

1 Universidad Federal de Sergipe (UFS), Aracaju-SE. * E-mail: wellington_life@live.com

${ }^{2}$ Centro Universitário AGES, Paripiranga-BA.

${ }^{3}$ Universidad Federal de Viçosa, Minas Gerais - MG

${ }^{4}$ Universidad Tiradentes (Unit), Aracaju-SE.

${ }^{5}$ Universidad Federal de Minas Gerais (UFMG), Minas Gerais-MG.

${ }^{6}$ Centro Universitário de Muriaé, Minas Gerais-MG

${ }^{7}$ Universidad Federal do Tocantins (UFT), Tocantins-MS 
of self-medication was among nursing technicians (5, 19.2\%) and nurses (4, 25\%). All professionals (45, $100 \%)$ are aware of the risks of self-medication. The drug most used by $(14,31.1 \%)$ of the professionals in the last 10 days was dipyrone. Conclusion: This indicates that there is a high rate of self-medication among health professionals.

Key words: Self-medication, Use of medication, Health professionals.

\section{RESUMO}

Objetivo: Alertar o público sobre o uso racional de medicamentos e os fatores associados a essa prática, destacando a importância de um profissional de saúde e promovendo ações preventivas. Método: Estudo descritivo, caráter exploratório e corte transversal. Estudos quantitativos de campo foram realizados na Unidade de Emergência e Maternidade Maria Jovita do Nascimento, em cinco unidades básicas de saúde, nos agentes de saúde da comunidade do Programa, no Centro de Vacinação e na Vigilância Epidemiológica em Fátima - BA. Os dados vieram de questionários semiestruturados para entender as opiniões individuais sobre o assunto em questão. O estudo foi aprovado pelo Comitê de Ética e Pesquisa com Seres Humanos do Centro Universitário AGES sob o Parecer № 024-2018. Resultados: Dos 45 (100\%) profissionais, (16, $35,5 \%)$ eram enfermeiros, (26, 57,8\%) eram técnicos de enfermagem e $(3,6,7 \%)$ eram auxiliares de enfermagem. A maior prevalência de automedicação foi entre técnicos de enfermagem $(5,19,2 \%)$ e enfermeiros (4, 25\%). Todos os profissionais (45, 100\%) estão cientes dos riscos da automedicação. 0 medicamento mais utilizado $(14,31,1 \%)$ dos profissionais nos últimos 10 dias foi a dipirona. Conclusão: Isso indica que há alta taxa de automedicação entre os profissionais de saúde.

Palavras-chaves: Automedicação, Uso de Medicamentos, Profissionais de Saúde.

\section{INTRODUCCIÓN}

De acuerdo con la Organización Mundial de la Salud (OMS), la automedicación es la selección y el uso de medicamentos por personas para tratar enfermedades y síntomas autodiagnosticados y debe ser entendida como uno de los elementos del autocuidado.

La automedicación ha sido muy frecuente a lo largo de los años, haciendo uso de fármacos sin prescripción, orientación y asesoría de un profesional de salud. La uso inadecuado de los medicamentos trae problemas, pues pueden perjudicar la salud del individuo. La problemática mencionada es ocasionada por el uso de fármacos sin prescripción médica, por iniciativa del propio paciente con el objetivo de aliviar los síntomas de la enfermedad. Además, existe en el mercado gran disponibilidad OTC's (Over the Counter), es decir los medicamentos que están disponibles en el estante de ventas, facilitando el acceso del cliente común a los fármacos (PAIM RSP, et al., 2016).

Dentro de los factores que permiten ese tipo de práctica están la dificultad al acceso por la populación a los servicios de salud y a la creencia de la necesidad de aliviar los síntomas. Y además, a la disponibilidad a los medicamentos aumenta la posibilidad del uso irracional (DOMINGUES PHF, et al., 2015).

Sin embargo esa práctica puede ser segura con algunos medicamentos restrictos y si los pacientes fueran orientados de manera adecuada por los farmacéuticos. El problema del uso irracional de medicamentos está en la falta de información de los riesgos, los efectos colaterales y las interacciones entre los medicamentos (OLIVEIRA AVC, et al., 2014).

El hecho de que los profesionales tengan acceso a los medicamentos facilita la automedicación. Además, su jornada de trabajo los condiciona para esa práctica equivocada, ya que la complexidad del trabajo, muchas veces el profesional tiene más de un empleo, pasan por situaciones estresantes y consecuentemente desarrollan alguna sintomatología, haciendo la automedicación un medio de facilitar su vida (BRITO EG, 2010).

REAS/EJCH | Vol. 11 (12) | e534 | DOI: https://doi.org/10.25248/reas.e534.2019 Página 2 de 7 
Aún se percibe que la falta de fiscalización en la salud pública permite un aumento de la automedicación, sin embargo existe otro lado de esta práctica, que es el hecho de ella ser económicamente más viable, ya que la disponibilidad de atención médica es muy precaria para todos los síntomas de la populación (luras A, et al., 2016). En vista de ello, la OMS, admite que, como esa práctica ha traído beneficios, una buena manera de fortalecer la populación de esa práctica es alertarlas mas, sobre la importancia del uso adecuado de los medicamento, en especial aquellos que son vendidos sin receta médica, teniendo así la necesidad de un profesional de la salud activo en la sociedad, promoviendo de ese modo la confianza, eficiencia y seguridad de los medicamentos administrado por el individuo (ARAÚJO AL, et al., 2015).

De hecho, los medicamentos son productos que, cuando se ingieren, pueden presentar beneficios, pero también pueden causar efectos no deseables para el consumidor. Concomitantemente, Matos (2005) resalta que la automedicación puede acarrear varios riesgos, como causar interacción medicamentosa; provocar efectos adversos y provocar un mayor riesgo, como la intoxicación; enmascarar enfermedades más graves, dificultando o retrasando las soluciones terapéuticas; interpretación incorrecta de los síntomas de la enfermedad y, consecuentemente, diagnósticos erróneos; la elección de un tratamiento farmacológico inadecuado desde el medicamento utilizado, la dosificación, la posología y la duración de la toma del mismo, entre otros.

El objetivo de este trabajo es llamar la atención de la población sobre el uso racional de los medicamentos, buscando demostrar los riesgos de los riegos de la automedicación, dando énfasis en tener un profesional de la salud para desarrollar acciones de prevención de la salud, identificando el perfil de los profesionales de salud que hacen uso de la automedicación y como principales clases medicamentosas utilizadas.

\section{MÉTODOS}

Se trata de un estudio descriptivo, de carácter investigativo, análisis de datos de sección transversal, con base en estudios en campo de carácter cuantitativo, entre marzo y septiembre de 2015, en la unidad de Urgencias y de Maternidad Maria Jovita do Nascimento, en cinco unidad básica de salud, en el Los trabajadores sanitarios de la comunidad Programa, en el Centro de Vacunas y en la Vigilancia Epidemológica en Fátima - BA, con una población de 17.652 habitantes.

Los datos recolectados fueron obtenidos de 45 profesionales de enfermería, siendo $(16 ; 35,6 \%)$ enfermeros, $(26 ; 57,8 \%)$ técnicos de enfermería y tres $(6,6 \%)$ auxiliares de enfermería, representando $100 \%$ de los profesionales de enfermería, que atendían los siguientes criterios: ser empleados con las Unidades, bajo el régimen de la Consolidación de las Leyes de Trabajo (CLT); tener realizado actividades de enfermería durante el tiempo que el investigador realizó el levantamiento de los datos y concordó de forma autónoma en participar de este estudio, firmando el Acuerdo de Consentimiento Libre y Esclarecido.

Los procedimientos utilizados para la colecta de datos partieron de cuestionarios semi-estructurados para entender las opiniones individuales sobre el tema en cuestión.

Los datos fueron obtenidos, analizados e interpretados de acuerdo con las siguientes variables, presentes en el cuestionario - datos personales, datos profesionales y datos de la investigación.

Los profesionales fueron captados a través de la ayuda de la coordinadora de la unidad basica de salud, en la que organizó a los profesionales en tres momentos para que los estudiantes de fármaco realizar las entrevistas a través de os cuestionarios.

Los procedimientos éticos fueron atendidos, de acuerdo con los requerimientos de la Resolución 466 del Consejo Nacional de Salud/Ministerio de la salud, el cual se refiere de investigaciones con seres humanos, la cual, sobre la visión del individuo, incorpora cuatro directrices básicas de la bio-ética: autonomía, beneficencia, no maleficencia y justicia.

El proyecto pasó por el comité de Ética e Investigación del Centro Universitario AGES bajo el dictamen $n^{\circ}$ 024-2018.

REAS/EJCH | Vol. 11 (12) | e534 | DOI: https://doi.org/10.25248/reas.e534.2019 Página 3 de 7 


\section{RESULTADOS Y DISCUSIÓN}

La automedicación se dá muchas veces porque las personas obtienen resultados y continúan tomando, ya que no hubo daños a la salud, sin embargo, el uso indiscriminado de esos fármacos puede traer consecuencias para el individuo cuando no hay acompañamiento médico o hasta una asesoría farmacéutica, siendo que esta práctica puede también desarrollar una enfermedad o perjudicar un tratamiento adecuado.

Se observó que profesionales de enfermería (29; 64,4\%) mencionaron haber tomado algún tipo de medicamento en los últimos 10 días, siendo que los auxiliares $(3 ; 100 \%)$ y los técnicos $(16 ; 61,5 \%)$ sobresalieron, seguido de los enfermeros (10;62,5\%). Cuando se indagó si el medicamento utilizado en los últimos 10 días fue por prescripción médica, la mayoría de las personas (20;44,4\%) respondió que sí, acompañado de los profesionales $(16 ; 35,6 \%)$ que no respondieron a esta pregunta y sólo $(9 ; 20,0 \%)$ respondieron que no, siendo que los técnicos de enfermería fue la categoría que sobresalió $(5 ; 19,2 \%)$ en la práctica de la automedicación, acompañados por los enfermeros (4; 25\%) (Tabla 1).

Fue observado que 29 profesionales de enfermería (64,4\%) afirmaron tomar algún tipo de medicamento en los últimos diez días, siendo que los tres auxiliares (100\%) y los 10 enfermeros $(62,5 \%)$ fueron sobresalientes en relación a los 16 técnicos $(61,5 \%)$. Cuando indagados si el medicamento utilizado en los últimos 10 días, la mayoría de las personas (44,4\%) afirmaron tener la prescripción médica, otras 16 (35,6\%) no respondieron a esta pregunta y apenas nueve (20\%) respondieron que no, siendo que, los 5 (19,2\%) técnicos de enfermería y 4 enfermeros (25\%) se sobresalieron en la práctica de automedicación (Cuadro 1).

Cuadro 1 - Distribución de los profesionales de enfermería según el uso de algún medicamento en los últimos diez días.

\begin{tabular}{|l|c|c|c|c|c|c|c|c|}
\hline \multicolumn{1}{|c|}{ PROFESIONALES } & \multicolumn{2}{c|}{ ENFERMERO } & \multicolumn{2}{c|}{ TEC. DE ENF. } & \multicolumn{2}{c|}{ AUX. DE ENF. } & \multicolumn{2}{c|}{ TOTAL } \\
\hline \multicolumn{1}{|c|}{ VARIÁBLES } & $\mathbf{N}$ & $\%$ & $\mathbf{N}$ & $\%$ & $\mathbf{N}$ & $\%$ & $\mathbf{N}$ & $\%$ \\
\hline Medicamento en los últimos diez días & \multicolumn{7}{|c|}{$\%$} & \multicolumn{4}{|c|}{} \\
\hline Si & 10 & 62,5 & 16 & 61,5 & 3 & $\mathbf{1 0 0 , 0}$ & 29 & 64,4 \\
\hline No & 6 & 37,5 & 10 & 38,5 & 0 & 0,0 & 6 & 35,6 \\
\hline TOTAL & $\mathbf{1 6}$ & $\mathbf{1 0 0 , 0}$ & $\mathbf{2 6}$ & $\mathbf{1 0 0 , 0}$ & $\mathbf{3}$ & $\mathbf{1 0 0 , 0}$ & $\mathbf{4 5}$ & $\mathbf{1 0 0 , 0}$ \\
\hline
\end{tabular}

Fuente: Datos recogidos por los autores, 2018.

De los profesionales que respondieron que el medicamento fue recetado los auxiliares de enfermería fue la categoría que sobresalió (3; 100\%), seguido de los técnicos $(11 ; 42,3 \%)$ y enfermeros $(6 ; 37,5 \%)$ (Figura 1).

Figura 1 - Relación de uso de medicamento y a receta médica.

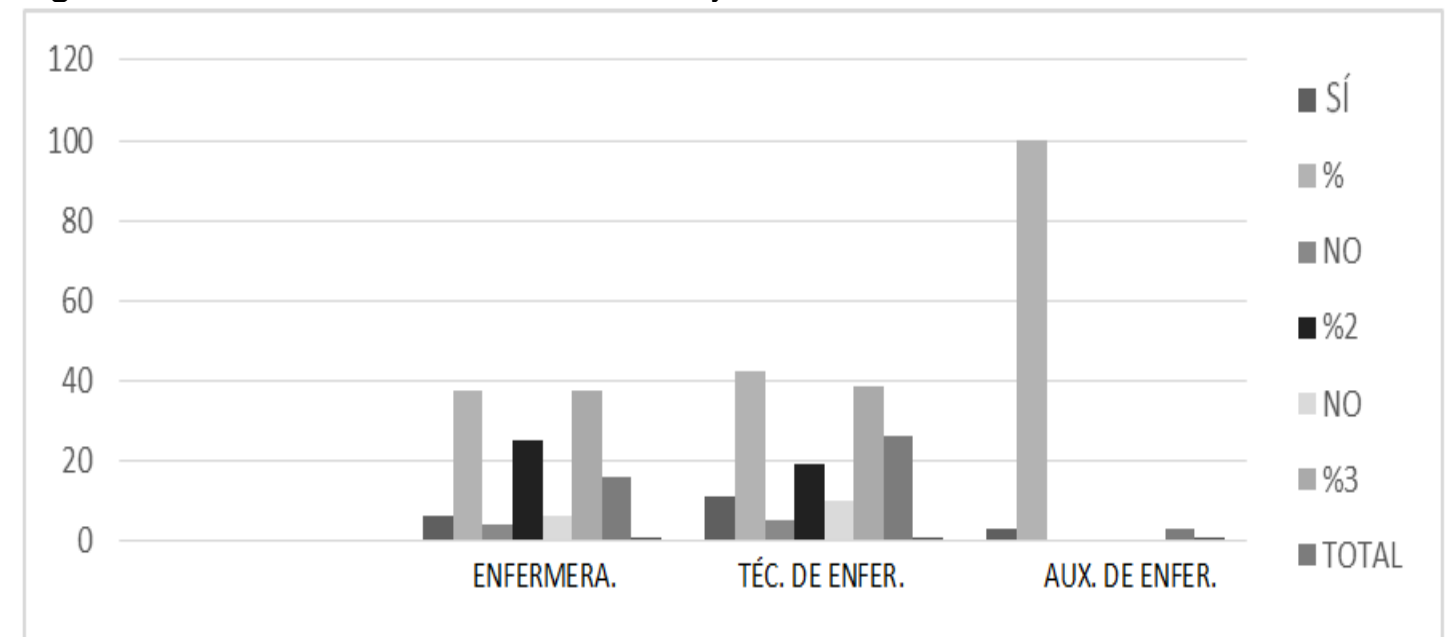

Fuente: Datos recogidos por los autores, 2018. 
La automedicación se mostró una práctica prevalente en la población estudiada. En Brasil, entre los profesionales de la enfermería, se identificaron los fármacos más comúnmente utilizados a través de la automedicación, siendo estos los de acción del sistema nervioso (46,7\%), aparato digestivo (15,4\%) y productos naturales en general $10 \%)$. En el caso de los analgésicos $(43,4 \%)$, el subgrupo más utilizado en este medio fue seguido por los antiinflamatorios y antirreumáticos $(7,3 \%)$ y vitaminas $(6,2 \%)$ (OLIVEIRA MA, et al., 2012).

La práctica de la automedicación estuvo más presente en trabajadores más jóvenes, lo que se asemeja a los resultados obtenidos en distintas poblaciones, o sea, la automedicación es inversamente proporcional a la edad. En este sentido, es posible aprender que la madurez evoca el sentido común de los profesionales y de las personas o, aunque las actuales generaciones de jóvenes están menos conscientes del cuidado de sí, arriesgando indebidamente a prácticas como la automedicación (CHEN J, et, al., 2014).

Fue unánime la respuesta de los profesionales, cuando indagados sobre el reconocimiento de los riesgos de la automedicación $(45 ; 100 \%)$. Sin embargo, teniendo conocimiento $(9 ; 20 \%)$ de esos profesionales aún practican la automedicación (Figura 2).

Figura 2 - Distribución de los trabajadores de enfermería, en relación al conocimiento de los riesgos de la automedicación.

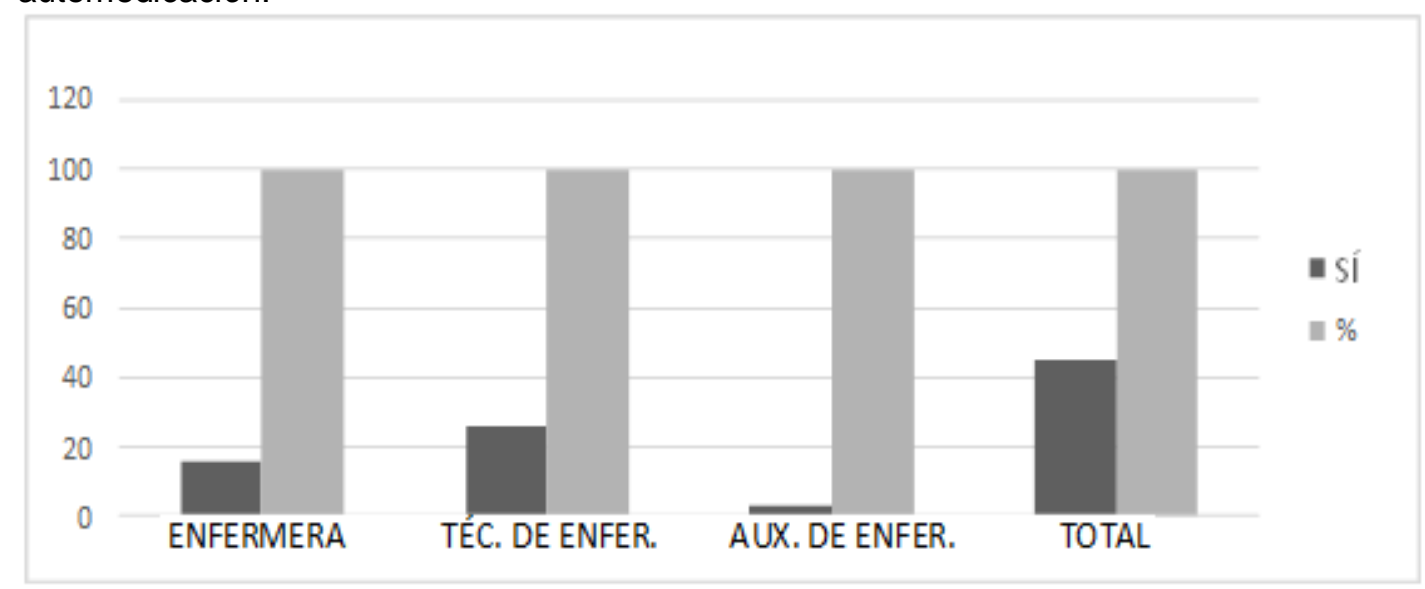

Fuente: Datos recogidos por los autores, 2018.

Pelos dados da pesquisa coletados, os principais medicamentos utilizados pelos profissionais nos últimos 10 dias de análise foram Dipirona (14; 31,1\%), Paracetamol (11;24,4\%), Ibuprofeno (9; 20\%), Prednisona (5; 11,1\%), Diazepam (3; 6,7\%) e Dorflex (3; 6,7\%) conforme Gráfico 3.

Con base en los datos obtenidos, los principales medicamentos utilizados por los profesionales en los últimos diez días de análisis fueron Dipirona (14;31,1\%), Paracetamol (11;24,4\%), Ibuprofeno (9; 20\%), Prednisona (5; 11,1\%), Diazepam (3; 6,7\%) e Dorflex (Citrato de Orfenadrina + Dipirona Sódica + Cafeína) (3; 6,7\%) como descrito en la (Figura 3).

En la investigación, los síntomas más citados para esta práctica de la automedicación fueron similares a los estudios en la población general, en que el dolor fue el síntoma que motivó la automedicación la mayoría de las veces (SHISHANI NF, 2014). Se destaca que el dolor de cabeza fue citado en todos los estudios seleccionados en esta investigación, lo que puede estar relacionado al estrés laboral de estos profesionales, teniendo en cuenta las responsabilidades peculiares y por liderar constantemente con el sufrimiento humano (GALATO D, 2012).

Entre los fármacos más consumidos están los AINE's (Anti-inflamatórios no estoroidales), todo eso para encontrar un alivio al dolor y esos medicamentos não necesitan de receta médica, siendo escogidos o por prescripción antigua o por sugerencia de terceros (vecinos) (SILVA JAC, et al., 2013). La sobrecarga de trabalho deja poco tiempo para los profesionales de enfermería promover el auto-cuidado, principalmente en 
lo que se refiere a la dificultad de adoptar un estilo de vida saludable, generando problemas de salud al profesional. Así, es posible afirmar que el trabajo de enfermería predispone al profesional a la práctica de auto-medicación en virtud de las características del trabajo y la carga horaria en exceso (PEREIRA RF, 2013).

Figura 3 - Distribución de los medicamentos usados en los últimos diez dias, por los profesionales.

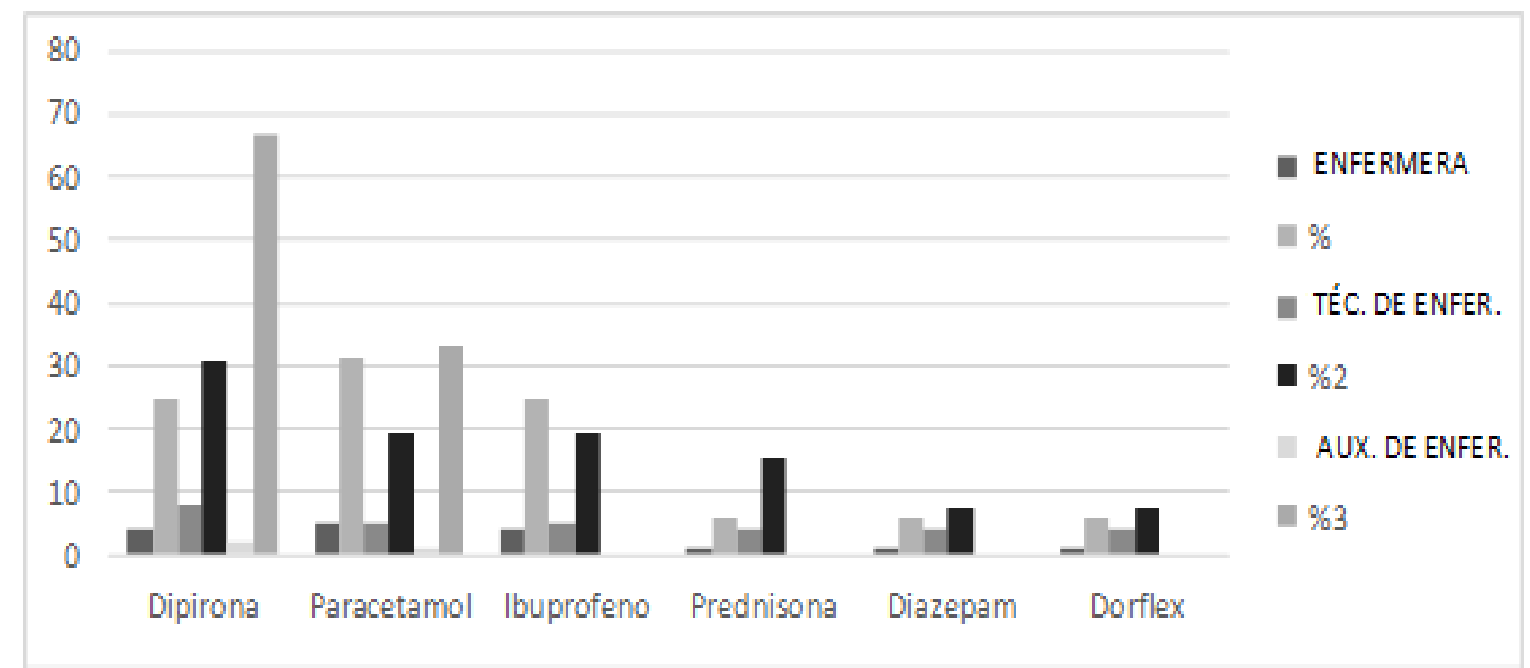

Fuente: Datos recogidos por los autores, 2018.

Delante de los cuestionamientos sobre cual es la razón que llevaron a estos profesionales a automedicarsen sin receta médica, fue el predominio de dolores, siendo la respuesta unánime $(45 ; 100 \%)$. Una vez que la auto-medicación entre los profesionales de enfermería dentro del ambiente hospitalario, muchas veces envuelven situaciones estresantes, de organización o sicológicos. En el cual pueden resultar en malestar, fatiga, somnolencia, insomnio, nauseas, irritaciones, perjuicios en la habilidad mental y desempeño (VIEIRA TG, et al., 2013).

Ya que, muchas de esas condiciones estresantes, hacen con que los trabajadores encuentren una posibilidad para facilitar la ejecución de su trabajo, en este caso, usando fármacos que minimicen esos factores, sin embargo siendo consientes de sus complicaciones que pueden causar (SOUZA LAF, et al., 2011). Pues, están pensando apenas en el momento para librarsen de la situación incómoda y no perciben que pueden llegar al abuso y dependencia.

Otro estudio desarrollado en España, la automedicación en situaciones de dolor en población mixta, (profesionales o no) fue practicada por 27,6\% de 1.964 participantes (con edades entre 20 y 91 años, de ambos sexos), contrastando con la prevalencia de 64\% observada entre los 270 clientes con dolores muscular y esquelética, de ambos sexos, que participaron en una investigación realizada en Temuco, Chile (TEJASHREE T, et al., 2014).

De esta manera los profesionales de enfermería están más susceptibles a manifestaciones o surgimiento de enfermedades físicas o sicológicas en virtud de la relación desgastante vinculada al trabajo. Por otro lado, el medio de trabajo permite el acceso a los medicamentos, lo que favorece el uso sin prescripción adecuada (SOUZA LAF, et al., 2011).

En ese sentido, aunque la automedicación tenga sus riesgos, esa práctica no puede ser considerada un acto estrictamente reprendido, pues muestra algunas ventajas como, por ejemplo, reducción del tempo, recursos y costos del tratamiento, para alivio de situaciones simples, dejando a los profesionales de la salud con mayor libertad para que se dediquen a situaciones clínicas mas delicadas e graves; reducción de los gastos del Estado con medicamentos, ya que esos gastos son en su totalidad pagos por los pacientes; aumento del lucro referente a la industria farmacéutica, entre otras (MORAIS ACC, 2011). 


\section{CONSIDERACIONES FINALES}

Al observar la automedicação entre los profesionales de enfermería, se encuentra una mayor prevalencia entre os técnicos de enfermería $(5 ; 19,2 \%)$ y los enfermeros $(4 ; 25 \%)$. Los resultados obtenidos mostraron que en los últimos 10 días el medicamento más utilizado por los profesionales fue Dopirona (14; $31,1 \%)$, siendo que por unanimidad los profesionales $(45 ; 100 \%)$ tomaron este medicamento con el intuito de aliviar dolores. Se conclue que la auto-medicación es un problema universal, antiguo y de grandes dimensiones. Aunque sea difícil evitar esa práctica, es imprescindible la orientación de la población ante el uso de medicamentos, sin estimular o consumo desmedido. En relación a los profesionales de enfermería, los efectos altamente peligrosos de los medicamentos puedes ser subestimados, siendo este un tema que debe ser abordado en los cursos preparatorios y en las estrategias direccionadas para mejorar de la salud de los profesionales.

\section{REFERENCIAS}

1. ARAÚJO AL, et al. Estudos brasileiros sobre automedicação: uma análise da literatura. Rev. Bras. Farm. 96 (2): $1178-1201,2015$.

2. BRITO ÉG. Automedicação dos Profissionais de Saúde: Uma Revisão de Literatura. Recife, 2010.

3. CHEN J, et al. A questionnaire-based survey study for the evaluation of knowledge of Pakistani university teachers regarding their awareness about ibuprofen as an over the counter analgesic. Acta Pol Pharm. 2014[citado em 2018 Nov.28];71(2):337-42.

4. DOMINGUES PHF, et al. Prevalência da automedicação na população adulta do Brasil: revisão sistemática. Revista Saúde Pública - v. 49 - nํ36, 2015.

5. GALATO D, et al. Self-medication among university studants: the influence of the field of study. Ciênc Saúde Coletiva. 2012 [citado em 2018 Nov. 28]; 17(12)3323-30.

6. IURAS A, et al. Prevalência da automedicação entre estudantes da Universidade do Estado do Amazonas (Brasil). Rev. Port. Estomatol. Med. Dente. cirmaxilofac. - v. 57 - nํ2, p. 104-111, 2016.

7. MORAIS ACC. Prevalência da Automedicação em Estudantes da Universidade de Aveiro. Departamento de Educação, 2011.

8. OLIVEIRA AVC, et al. Falência hepática aguda e automedicação. ABCD Arq. Bras. Cir. Dig. - V. 27 - no 4 -, p.294297, 2014.

9. OLIVEIRA MA, FRANCISCO PMSB, COSTA KS, BARROS MBA. Self-medication in the elderly population of Campinas, São Paulo State, Brazil: prevalence and associated factors. Cad Saúde Pública. 2012[citado em 2018 Nov.28];28(2):335-45.

10. PAIM RSP, et al. Automedicação: uma síntese das publicações Nacionais. Revista Contexto \& Saúde, ljuí: Editora Unijuí, - v. 16 - no 30, p. 47-54, 2016.

11. PEREIRA RF. Fatores de Risco Cardiovascular em Trabalhadores de Enfermagem de um Hospital Público em São José dos Campos-SP, 2013.

12. SHISHANI NF, et al. Medication self-administered behavior among Jordanian population. RMJ. 2014 [citado em 2018 Nov.28];39(1):35-8.

13. SILVA JAC, et al. Prevalência de automedicação e os fatores associados entre os usuários de um Centro de Saúde Universitário. Rev. Bras. Clin. Med. São Paulo, jan-mar;11(1):27-30, 2013.

14. SOUZA LAF, et al. Prevalência e caracterização da prática de automedicação para alívio da dor entre estudantes universitários de enfermagem. Rev. Latino-Am. Enfermagem, - v. $19-\mathrm{n}^{\circ}$ 2, 2011.

15. TEJASHREE $\mathrm{T}$, et al. Evaluation of self-medication practices among medical and non-medical individuals. Indian $\mathrm{J}$ Med Res Pharm Sci. 2014[citado em 2018 Nov.28];5(1):212-8.

16. VIEIRA TG, et al. Illness and the use of psychoactive drugs among nursing workers at intensive care units. Rev. Enferm UFSM. 2013[citado em 2018 Nov. 28];3(2):205-14. 